Pacific Journal of Mathematics

GLÄTTUNGEN VON ABBILDUNGEN 3-DIMENSIONALER 


\title{
GLÄTTUNGEN VON ABBILDUNGEN 3-DIMENSIONALER MANNIGFALTIGKEITEN
}

\author{
HARALD BOEHME
}

\begin{abstract}
Let $N$ and $M$ be compact 3-dimensional manifolds, $f: N \rightarrow M$ a map. Furthermore let given a Heegaard splitting for $M$, which certainly exists if $M$ is closed, and then it is a pair $(V, W)$, where $V, W$ are handlebodies (possibly non orientable) with $M=V \cup W, V \cap W=\partial V=\partial W$. It will be shown, that $f$ can be deformed into a normal form with respect to the Heegaard splitting of $M$ : either $f$ has the absolute degree 0 , or $f$ is homotopic to a map $g$ with the property that $g \mid g^{-1}(V)$ is a covering map.
\end{abstract}

Einen ersten Spezialfall des hier vorgeführten Resultats erhielt E. Moise im Zusammenhang mit der Poincaréschen Vermutung [6], [7]. In seinem Theorem ist die Aussage enthalten, daß eine Abbildung der 3-Sphäre $f: S^{3} \rightarrow M$ mit grad $f=1$ homotop ist zu einer Abbildung $g$, wobei $g \mid g^{-1}(V)$ ein Homöomorphismus und $V$ ein Henkelkörper zu einer gegebenen Heegaard-Zerlegung von $M$ ist (das gleiche gilt auch für W. Haken [2]). Dieses Ergebnis wurde von $\mathrm{F}$. Waldhausen wesentlich verschärft und zugleich auf beliebige Abbildungen von geschlossenen, orientierbaren 3-dimensionalen Mannigfaltigkeiten mit grad $f=1$ ausgedehnt [9].

Nach diesen Arbeiten entstand die Frage, ob sich auch für Abbildungen mit beliebigem Abbildungsgrad eine Normalform finden läßt. Dies ist tatsächlich der Fall, und ergibt sich aus einer Verallgemeinerung der alten Methode von H. Kneser zur Glättung von Flächenabbildungen [4], [5]. Er ging dabei von simplizialen Abbildungen aus und betrachtete die Urbilder der einzelnen Simplexe. Es ist klar, daß diese für die Codimensionen 0 und 1 nicht sehr kompliziert sein können; die entscheidenden Singularitäten sind "Falten", d.h. zwei benachbarte 2-Simplexe werden auf eins abgebildet. Eine solche Falte verschwindet durch eine einfache Deformation der gemeinsamen Grundseite, jedoch muß H. Kneser dazu noch geeignete simpliziale Unterteilungen angeben. Die adäquate Beschreibung der geometrischen Idee gelingt erst in der semilinearen Kategorie. Dazu wérden die Simplexe durch Henkel ersetzt und die Glättungen über den 0- und 1-Henkeln durchgeführt, welche zusammen einen Henkelkörper $V$ bilden. Für eine Abbildung 3dimensionaler Mannigfaltigkeiten $f: N \rightarrow M$ ist nach Beseitigung der Falten $f \mid f^{-1}(V)$ schon eine Überlagerung auf allen wesentlichen Komponenten. Das Problem, diese Komponenten durch eine Defor- 
mation von $f \mathrm{zu}$ vereinigen, kann gelöst werden, falls $V$ von einer Heegaard-Zerlegung von $M$ kommt.

o. Notation. Es wird in der semilinearen Kategorie gearbeitet. Alle vorkommenden Mannigfaltigkeiten sind kompakt. Reguläre Umgebungen sind klein gegenüber allen anderen Objekten in der Mannigfaltigkeit. Ein $n$-Element $E^{n}$ ist homöomorph dem $n$-Simplex.

Sei $M$ eine 3-dimensionale Mannigfaltigkeit.

Sei $J$ eine Mannigfaltigkeit in $M$, nicht notwendig zusammenhängend, $J \cap \partial M=\partial J$, codim $J=q$. Eine Umgebung $V(J)$ in $M$ ist prismatisch, wenn sie sich mit einem Bündel $J \times E^{q}$ über $J$ identifizieren läßt, so daß $J=J \times x_{0}, x_{0} \in \stackrel{\circ}{E}^{q}$, und $\left(J \times E^{q}\right) \cap \partial M=\partial J \times E^{q}$.

Sei $\Gamma$ ein zusammenhängender Graph in $M, \Gamma \cap \partial M=\varnothing$. Eine reguläre Umgebung $V(\Gamma)$ in $M$ ist ein Henkelkörper in $M$. $\mathrm{Er}$ besteht aus einer prismatischen Umgebung $V\left(\Gamma^{0}\right)$ in $M\left(\Gamma^{0}\right.$ ist die Menge der Ecken von $\Gamma$ ), das sind 3-Elemente $h_{1}^{0}, \cdots, h_{s}^{0}$, und aus einer prismatischen Umgebung $V\left(\left(\Gamma-V\left(\Gamma^{0}\right)\right)^{-}\right)$in $\left(M-V\left(\Gamma^{0}\right)\right)^{-}$, das sind 3 -Elemente $h_{1}^{1}, \cdots, h_{t}^{1}$. Dabei ist $h^{r}$ ein $r$-Henkel, und es ergibt sich insgesamt eine Henkelzerlegung von $V(\Gamma)$, siehe [3].

1. Faltenreduktion. Seien $N$ und $M$ 3-dimensionale Mannigfaltigkeiten, sei $f:(N, \partial N) \rightarrow(M, \partial M)$ eine Abbildung.

Definition. Sei $J$ eine Mannigfaltigkeit in $M, J \cap \partial M=\partial J . \quad f$ ist transversal bzgl. $J$, wenn es eine prismatische Umgebung $V(J)$ in $M$ gibt, so daß $f^{-1}(V(J))$ ein Bündel ist, und $f$ jede Faser homöomorph auf eine Faser von $V(J)$ abbildet.

Definition. Sei $\Gamma$ ein Graph in $M, \Gamma \cap \partial M=\varnothing . \quad f$ ist transversal bzgl. $\Gamma$, wenn gilt:

(1) $f$ ist transversal bzgl. $\Gamma^{0}$; die zugehörige prismatische Umgebung sei $V\left(\Gamma^{0}\right)$.

(2) Die von $f$ induzierte Abbildung

$$
\left(N-f^{-1}\left(V\left(\Gamma^{0}\right)\right)\right)^{-} \longrightarrow\left(M-V\left(\Gamma^{0}\right)\right)^{-}
$$

ist transversal bzgl. $\left(\Gamma-V\left(\Gamma^{0}\right)\right)^{-}$.

Sei $f$ transversal bzgl. $\Gamma$, dann ist $f^{-1}\left(\Gamma^{0}\right)$ eine Menge von Ecken in $N$, ihre Anzahl sei $\alpha\left(f, \Gamma^{0}\right)$, und $f^{-1}(\Gamma)$ ist ein 1-dimensionaler Komplex in $N$, die Anzahl der Komponenten sei $\beta(f, \Gamma)$. Das Tupel $(\alpha, \beta)$ in lexicographischer Ordnung mißt die Kompliziertheit von $f$ bzgl. $\Gamma$.

Sei $\Lambda$ eine Komponente von $f^{-1}(\Gamma)$, sei $\Lambda^{0}=\Lambda \cap f^{-1}\left(\Gamma^{0}\right)$. Es gilt genau einer der folgenden Fälle: 
(1) $\Lambda^{0} \neq \varnothing ; \Lambda$ wird dann als Graph bezeichnet, wobei $\Lambda^{0}$ die Menge der Ecken ist.

(2) $\Lambda^{0}=\varnothing$; in diesem Fall ist $\Lambda$ ein Zyklus.

Lemma 1.1. Seien $N$ und $M$ 3-dimensionale Mannigfaltigkeiten, sei $\Gamma$ ein zusammenhängender Graph in $M, \Gamma \cap \partial M=\varnothing . \quad$ Sei $f:(N, \partial N) \rightarrow(M, \partial M)$ eine Abbildung, dann gibt es eine zu $f$ homotope Abbildung g, so da $\beta$ gilt:

(1) $g$ ist transversal bzgl. $\Gamma$.

(2) Für jede Komponente $\Lambda$ von $g^{-1}(\Gamma)$, die ein Graph ist, ist $g \mid \Lambda: \Lambda \rightarrow \Gamma$ eine Überlagerung.

Beweis. (1) Nach einer Homotopie analog [8, 1.1] ist $f$ transversal bzgl. $\Gamma^{0}$, die weitere Transversalität läßt sich ebenso erreichen. Die entstehende Abbildung sei $g$, und die zugehörige reguläre Umgebung $V(\Gamma)$ habe die Henkelzerlegung

$$
V(\Gamma)=\bigcup_{i=1}^{s} h_{i}^{0} \cup \bigcup_{j=1}^{t} h_{j}^{1} .
$$

(2) Sei $\Lambda$ eine Komponente von $g^{-1}(\Gamma)$, die ein Graph ist. Um $g \mid \Lambda \mathrm{zu}$ einer Überlagerung $\mathrm{zu}$ machen, genügt es, wenn für jede Kante $l$ von $\Lambda$ mit $g(l)=k, k$ ist eine Kante von $\Gamma$, die induzierte Abbildung $\left(l^{\prime}, \partial l^{\prime}\right) \rightarrow\left(k^{\prime}, \partial k^{\prime}\right)$ den Grad \pm 1 hat, wobei

$$
l^{\prime}=\left(l-g^{-1}\left(\bigcup_{i=1}^{s} h_{i}^{0}\right)\right)^{-}, k^{\prime}=\left(k-\bigcup_{i=1}^{s} h_{i}^{0}\right)^{-} .
$$

Eine Kante $l$ von $\Lambda$, die das nicht erfüllt, ist eine Falte von $g$ über $k$ ([4], $\Gamma$ entspricht dem dualen 1-Gerüst dort).

Sei $l$ eine Falte, seien $U_{i}, i=1,2$, Komponenten von $g^{-1}\left(\bigcup_{i=1}^{s} h_{i}^{0}\right)$, sei $U_{3}$ eine Komponente von $g^{-1}\left(\bigcup_{j=1}^{t} h_{j}^{1}\right)$, so daß $U=\bigcup_{i=1}^{2} U_{i} \cup U_{3}$ eine reguläre Umgebung von $l$ ist. Es gibt eine Homotopie von $g$ rel $g^{-1}\left(\bigcup_{i=1}^{s} h_{i}^{0}\right)$, deren Abbildungen transversal bzgl. $\Gamma$ sind, $g^{-1}(\Gamma)$ fest lassen, und die selben zugehörigen Umgebungen haben, so da $B$ $g\left(l^{\prime}\right)=P, \quad P \in \partial k^{\prime}$. Sei ferner $P \in \partial h_{1}^{0}$, dann induziert jetzt $g$ die Abbildung $g \mid U:(U, \partial U) \rightarrow\left(h_{1}^{0}, \partial h_{1}^{0}\right)$. Da $P \notin g(\partial U)$, gibt es eine Homotopie von $g \mid U$ rel $\partial U$, so daß $g(U) \subset \partial h_{1}^{0}$. Danach hat $g^{-1}\left(\Gamma^{0}\right)$ genau zwei Ecken weniger. Die Transversalität von $g$ bzgl. $\Gamma$ wird wieder hergestellt, ohne $g^{-1}\left(\Gamma^{0}\right)$ zu ändern, für die neue Kompliziertheit gilt dann $\alpha^{\prime}=\alpha-2$. Nach endlich vielen Schritten sind alle Falten beseitigt.

2. Abbildungen über Heegaard-Zerlegungen.

Definition. Sei $M$ eine 3-dimensionale Mannigfaltigkeit. Eine 
Heegaard-Zerlegung von $M$ ist ein geordnetes Paar von Untermannigfaltigkeiten $(V, W)$, so $\operatorname{da} B \quad M=V \cup W, V \cap W=\partial V=\partial_{-} W$, $\partial_{-} W$ ist eine Komponente von $\partial W$, und es gilt:

(1) $V$ ist ein Henkelkörper in $M$.

(2) $W$ hat eine Henkelzerlegung

$$
W=\partial_{-} W \times I \cup \bigcup_{k=1}^{u} h_{k}^{2} \cup \bigcup_{l=1}^{v} h_{l}^{3},
$$

$\partial_{-} W=\partial_{-} W \times 0$

Das Geschlecht der Heegaard-Zerlegung ist das Geschlecht von $V$.

Sei $\partial M=\varnothing$, dann besitzt $M$ sicher eine Heegaard-Zerlegung, und $W$ ist ebenfalls ein Henkelkörper in $M$.

SATz 2.1. Seien $N$ und $M$ 3-dimensional Mannigfaltigkeiten, sei $(V, W)$ eine Heegaard-Zerlegung von $M$, deren Geschlecht $\geqq 1$ ist, ferner sei $\Gamma$ ein Graph in $M$ und $V$ eine reguläre Umgebung von $\Gamma$. Sei $f:(N, \partial N) \rightarrow(M, \partial M)$ eine Abbildung, dann gibt es eine zu $f$ homotope Abbildung $g$, so daß entweder (a) oder (b) gilt.

(a) $\alpha\left(g, \Gamma^{0}\right)=0$

(b) $g \mid g^{-1}(V): g^{-1}(V) \rightarrow V$ ist eine Überlagerung.

Beweis. Zunächst wird $f$ in eine Abbildung $g$ deformiert, für die (1.1) bzgl. $\Gamma$ gilt. Es kann angenommen werden, daß $V$ eine Umgebung von $\Gamma$ zur Transversalität von $g$ ist, und $V$ habe die Henkelzerlegung $V=\bigcup_{i=1}^{s} h_{i}^{0} \cup \bigcup_{j=1}^{t} h_{j}^{1}$. Es folgt Fall (b), wenn $g^{-1}(\Gamma)$ ein zusammenhängender Graph ist.

Sei $\alpha\left(g, \Gamma^{0}\right) \neq 0$, dann gibt es eine Komponente $\Lambda_{1}$ von $g^{-1}(\Gamma)$, die ein Graph ist, und $g \mid \Lambda_{1}: \Lambda_{1} \rightarrow \Gamma$ ist eine Überlagerung. Sei $\Lambda_{2}$ eine weitere Komponente von $g^{-1}(\Gamma), \Lambda_{2}$ ist ein Graph oder ein Zyklus. Im ersten Fall hat $\Lambda_{2}$ das Geschlecht $\geqq 1$, also gibt es in $\Lambda_{2}$ eine nicht zerlegende Kante $l_{2}$. Im zweiten Fall sei $\Lambda_{2}=l_{2}$ Sei $k$ die Kante von $\Gamma$ mit $g\left(l_{2}\right) \subset k$, und sei $h_{1}^{1}$ der zugehörige 1-Henkel von $V$. Sei $Q_{2} \in l_{2} \cap g^{-1}\left(h_{1}^{1}\right), g\left(Q_{2}\right)=P$, ferner seien $Q_{1,1}, \cdots, Q_{1, n}$ die Punkte in $\Lambda_{1}$ mit $\mathrm{g}\left(Q_{1, p}\right)=P, p=1, \cdots, n$. Sei $P^{\prime} \in \partial h_{1}^{1}$ in der Faser über $P, Q_{1, p}^{\prime} b z w$. $Q_{2}^{\prime}$ seien Punkte in der Faser über $Q_{1, p} b z w . Q_{2}$ mit $g\left(Q_{1, p}^{\prime}\right)=g\left(Q_{2}^{\prime}\right)=P^{\prime}$.

LEMMA 2.2. Es gibt eine Homotopie von $g$ rel $g^{-1}(V)$, wonach in $g^{-1}(W)$ ein Weg $w$ von $Q_{1, p}^{\prime}$ nach $Q_{2}^{\prime}$ existiert, $p \in\{1, \cdots, n\}$, so da $\beta$ die von $g$ induzierte Abbildung $(w, \partial w) \rightarrow\left(W, P^{\prime}\right)$ nullhomotop ist.

Beweis. In $g^{-1}(W)$ gibt es einen Weg $w$ von $Q_{1,1}^{\prime}$ nach $Q_{2}^{\prime}$. Durch eine Homotopie von $g \operatorname{rel} g^{-1}(V)$ läßt sich $g(w)$ aus $\bigcup_{l=1}^{v} h_{l}^{3}$ 
und $\bigcup_{k=1}^{u} h_{k}^{2}$ herausdrücken, es ist dann $g(w) \subset\left(\partial_{-} W \times I\right)-\left(\partial_{-} W \times 1\right)$. Nach einer weiteren Homotopie von $g$ rel $g^{-1}(V)$ ist $g$ transversal bzgl. $\left(\left(\partial_{-} W \times 1\right)-\partial W\right)^{-}$, und $g^{-1}\left(\partial_{-} W \times I\right)$ ist eine Untermannigfaltigkeit in $N$, welche $w$ enthält.

In $\partial_{-} W$ sei das System $c_{1}, \cdots, c_{m}$ von einfachen Kurven eine kanonische Zerschneidung mit dem gemeinsamen Punkt $c_{0}$, ferner sei $C_{i}=c_{i} \times I, \quad i=0, \cdots, m$. Nach weiteren Homotopien ist $g \mid g^{-1}\left(\partial_{-} W \times I\right)$ transversal bzgl. $C_{0}, \cdots, C_{m}$, d.h.

(1) $g \mid g^{-1}\left(\partial_{-} W \times I\right)$ ist transversal bzgl. $C_{0}$; die zugehörige prismatische Umgebung sei $U_{0}$.

(2) Die von $g$ induzierte Abbildung

$$
g^{-1}\left(\left(\left(\partial_{-} W \times I\right)-U_{0}\right)^{-}\right) \longrightarrow\left(\left(\partial_{-} W \times I\right)-U_{0}\right)^{-}
$$

ist transversal bzgl. $\left(C_{1}-U_{0}\right)^{-}, \cdots,\left(C_{m}-U_{0}\right)^{-}$.

Sei $F_{i}=g^{-1}\left(\left(C_{i}-U_{0}\right)^{-}\right), \quad \mathrm{i}=1, \cdots, m$, dies ist eine Fläche in $\left.g^{-1}\left(\left(\partial_{-} W \times I\right)-U_{0}\right)^{-}\right)$, nicht notwendig zusammenhängend. In allgemeiner Lage ist auch $w$ in $g^{-1}\left(\left(\left(\partial_{-} W \times I\right)-U_{0}\right)^{-}\right)$, und $w$ trifft die Flächen $F_{1}, \cdots, F_{m}$ transversal. Von $Q_{1,1}^{\prime}$ ausgehend werde zunächst $F_{1}$ getroffen, dieser erste Teilweg sei $w_{1} \cdot g\left(w_{1}\right)$ zeichnet eine Seite von $C_{1}$ aus, und damit auch von $c_{1}$. $\mathrm{Zu}$ dieser Seite läßt sich $P^{\prime}$ mit $c_{1}$ durch einen $\mathrm{Weg}$ in $\partial_{-} W$ verbinden, ohne daß die kanonische Zerschneidung sonst getroffen wird. Die Liftung davon ergibt einen Weg $w_{0}$ in $g^{-1}\left(\partial_{-} W\right)$ von $Q_{1,1}^{\prime}$ nach $F_{1}$. Der Weg $w_{0} \cup w_{1}$ wird leicht von $g^{-1}\left(\partial_{-} W\right)$ abgehoben, es entsteht ein Weg $w^{\prime}$ mit $w^{\prime} \cap F_{i}=\partial w^{\prime}, i=1$, und $w^{\prime} \cap F_{i}=\varnothing$ sonst.

Aus der Konstruktion folgt, daß die von $g$ induzierte Abbildung $\left(w^{\prime}, \partial w^{\prime}\right) \rightarrow\left(\partial_{-} W \times I, C_{1}\right)$ nullhomotop ist. Ohne die Situation sonst zu ändern gibt es eine Homotopie von $g \mid g^{-1}\left(\partial_{-} W \times I\right)$, deren Abbildungen transversal bzgl. $C_{0}, \cdots, C_{m}$ sind, so daß $g\left(w^{\prime}\right) \subset I$, wobei $I$ eine Faser der zugehörigen prismatischen Umgebung von $\left(C_{1}-U_{0}\right)^{-}$ ist. Dann läßt sich $g$ so deformieren, daß in $F_{1}$ eine reguläre Umgebung von $\partial w^{\prime}$ durch einen Kreisring ersetzt wird, der in einer regulären Umgebung von $w^{\prime}$ liegt und $w \cup w^{\prime}$ nicht trifft (dies ist die Umkehrung zu [8, S. $507 u$. $]$ ). Das nunmehr freie Ende von $w^{\prime}$ bei $g^{-1}\left(\partial_{-} W\right)$ wird mit einem Punkt $Q_{1,1}^{\prime \prime}$ aus $g^{-1}\left(\partial_{-} W\right)$ verbunden. In $\partial_{-} W$ gibt es einen Weg von $g\left(Q_{1,1}^{\prime \prime}\right)$ nach $P^{\prime}$, der die kanonische Zerschneidung sonst nicht trifft. Die Liftung davon ergibt einen Weg $w^{\prime \prime}$ in $g^{-1}\left(\partial_{-} W\right)$ von $Q_{1,1}^{\prime \prime}$ nach $Q_{1, p}^{\prime}, p \in\{1, \cdots, n\}$. In $w$ wird $w_{1}$ durch $w^{\prime} \cup w^{\prime \prime}$ ersetzt, der neue Weg trifft $F_{1}, \cdots, F_{m}$ in einem Punkt weniger. Schließlich ist $\mathrm{w} \cap F_{i}=\varnothing$, und damit $g(w) \cap C_{i}=\varnothing$, $i=1, \cdots, m$.

Sei $w$ der Weg aus (2.2), es gibt dann eine Homotopie von $g$ rel $g^{-1}(V)$ so daß $g(w)=P^{\prime}$. In einer Faser von $\mathrm{h}_{1}^{1}$ wird $P$ durch 
einen einfachen Weg mit $P^{\prime}$ verbunden, in den Fasern über $Q_{1, p}$ bzw. $Q_{2}$ sind die Urbilder dazu Wege nach $Q_{1, p}^{\prime}$ bzw. $Q_{2}^{\prime}$, insgesamt entsteht ein einfacher Weg $\widetilde{w}$ von $Q_{1, p}$ nach $Q_{2}$. Nun läßt sich $g$ so deformieren, daß in $g^{-1}(\Gamma)$ eine reguläre Umgebung von $\partial \widetilde{w}$ durch zwei Wege längs $\widetilde{w}$ ersetzt wird, $g$ wieder transversal bzgl. $\Gamma$ ist, und alle anderen Objekte fest bleiben. Danach hat $g^{-1}(\Gamma)$ eine Komponente weniger, für die neue Kompliziertheit gilt $\alpha^{\prime}=\alpha, \beta^{\prime}=\beta-1$. Es ist nun (1.1) erfüllt, oder die Beseitigung von Falten ergibt $\alpha^{\prime \prime}<\alpha$. Die Abbildung ist also so lange $\mathrm{zu}$ vereinfachen, bis die Behauptung von (2.1) gilt.

Korollar 2.3 Seien $N$ und $M$ geschlossene 3-dimensionale Mannigfaltigkeiten, sei $(V, W)$ eine Heegaard-Zerlegung von $M$, deren Gechlecht $\geqq 1$ ist. Sei $f: N \rightarrow M$ eine Abbildung, dann gibt es eine zu $f$ homotope Abbildung $g$, so daß entweder (a) oder (b) gilt.

(a) $g(N) \subset K^{2} ; K^{2}$ ist ein 2-dimensionaler Komplex in $M$.

(b) In $N$ gibt es Untermannigfaltigkeiten $X, Y, X \cap Y=\varnothing$, so $\quad d a ß \quad g|X: X \rightarrow V, \quad g| Y: Y \rightarrow W$ Überlagerungen sind und $g(N-(X \cup Y))^{-} \subset \partial V$.

Beweis. Seien $\Gamma$ bzw. $\Gamma_{1}$ Graphen in $M$, so daß $V$ bzw. $W$ reguläre Umgebungen davon sind. Sei $W_{1}$ eine reguläre Umgebung von $\Gamma_{1}$ in $W$, es ist dann $\left(W-W_{1}\right)^{-}=\partial W \times I, \partial W=\partial W \times 0$, und damit $\left(W_{1}, \partial W \times I\right)$ eine Heegaard-Zerlegung von $W$. Nach (2.1) gibt es eine $\mathrm{zu} f$ homotope Abbildung $g$, für die entweder (a) oder (b) gilt.

(a) $g(N) \subset \bigcup_{j=1}^{t} h_{j}^{1} \cup W$

(b) $g \mid g^{-1}(V): g^{-1}(V) \rightarrow V$ ist eine Überlagerung.

Im Fall (a) läßt sich $g(N)$ auf einen Komplex $K^{2}$ retrahieren. Im Fall (b) wird (2.1) wieder für die Abbildung $g \mid g^{-1}(W): g^{-1}(W) \rightarrow W$ angewandt, dabei kann die Homotopie natürlich auf dem Rand konstant gewählt werden. Es entsteht eine $z u f$ homotope Abbildung $g_{1}$, für die entweder $\left(a_{1}\right)$ oder $\left(b_{1}\right)$ gilt.

( $\left.\mathrm{a}_{1}\right) \quad g_{1}(N) \subset V \cup \partial W \times I \cup \bigcup_{k=1}^{u} h_{k}^{2}$

(b) $g_{1} \mid g_{1}^{-1}\left(W_{1}\right): g_{1}^{-1}\left(W_{1}\right) \rightarrow W_{1}$ ist eine Überlagerung.

Im Fall $\left(a_{1}\right)$ läßt sich wieder $g_{1}(N)$ auf einen Komplex $K_{1}^{2}$ retrahieren. Im Fall $\left(b_{1}\right)$ sei $X=g_{1}^{-1}(V), Y=g_{1}^{-1}\left(W_{1}\right)$, damit ist $g_{1}(N-(X \cup Y))^{-} \subset \partial W \times I$. Es gibt eine zur Identität homotope Abbildung $p:\left(W_{1}, \partial W \times I\right) \rightarrow(W, \partial V)$, wobei $p \mid W_{1}: W_{1} \rightarrow W$ ein Homöomorphismus ist und $p \mid \partial W \times I$ die Projektion auf $\partial W=\partial V$. Mit $g=p g_{1}$ folgt die Behauptung (b). 
Bemerkung. In (2.1) sei $A$ der Absolutgrad von $f$. Sei $z \in \Gamma^{0}$, dann gibt es eine zu $f$ homotope Abbildung $g$, so daß $g$ transversal bzgl. $z$ ist, und $g^{-1}(z)$ genau $A$ Komponenten hat [1]. Im Beweis von (2.1) kann sich diese Anzahl höchstens durch Faltenreduktion verringern, was aber nicht möglich ist. Danach ergibt sich Fall (a) genau dann, wenn $A=0$, und im Fall (b) ist $A$ die Blätterzahl von $g \mid g^{-1}(V)$.

\section{LITERATUR}

1. D. B. A. Epstein, The degree of a map, Proc. London Math. Soc., (3) 16 (1966), 369-383.

2. W. Haken, On homotopy 3-spheres, Illinois J. Math., 10 (1966) 159-178.

3. J. F. P. Hudson, Piecewise linear topology, New York. Benjamin 1969.

4. H. Kneser, Glättungen von Flächenabbildungen, Math. Ann., 100 (1928), 609-617.

5. — Die kleinste Bedeckungszahl innerhalb einer Klasse von Flächenabbildungen, Math. Ann., 103 (1930), 347-358.

6. E. Moise, Simply connected 3-manifolds. Topology of 3-manifolds and related topics, Prentice-Hall 1962.

7. - A monotonic mapping theorem for simply connected 3-manifolds, Illinois J. Math., 12 (1968), 451-474.

8. F. Waldhausen, Gruppen mit Zentrum und 3-dimensionale Mannigfaltigkeiten, Topology, 6 (1967), 505-517.

9. On mappings of handlebodies and of Heegard splittings, Topology of manifolds, Chicago, Markham 1970.

Received July 25, 1974.

Freie Universität Berlin-West 



\section{PACIFIC JOURNAL OF MATHEMATICS}

\section{EDITORS}

RICHARD ARENS (Managing Editor) University of California

Los Angeles, California 90024

\section{R. A. BEAUMONT}

University of Washington Seattle, Washington 98105
J. DugundjI Department of Mathematics University of Southern Californı Los Angeles, California 90007

D. Gilbarg AND J. Milgram Stanford University Stanford, California 94305

\section{ASSOCIATE EDITORS}

E. F. BECKENBACH

B. H. NeUMANN

F. WOLF

K. YosHidA

\section{SUPPORTING INSTITUTIONS}

UNIVERSITY OF BRITISH COLUMBIA CALIFORNIA INSTITUTE OF TECHNOLOGY UNIVERSITY OF CALIFORNIA MONTANA STATE UNIVERSITY UNIVERSITY OF NEVADA NEW MEXICO STATE UNIVERSITY OREGON STATE UNIVERSITY UNIVERSITY OF OREGON OSAKA UNIVERSITY

\author{
UNIVERSITY OF SOUTHERN CALIFORNIA \\ STANFORD UNIVERSITY \\ UNIVERSITY OF TOKYO \\ UNIVERSITY OF UTAH \\ WASHINGTON STATE UNIVERSITY \\ UNIVERSITY OF WASHINGTON \\ AMERICAN MATHEMATICAL SOCIETY \\ NAVAL WEAPONS CENTER
}




\section{Pacific Journal of Mathematics}

\section{Vol. 61, No. $1 \quad$ November, 1975}

Jiří Adámek, V. Koubek and Věra Trnková, Sums of Boolean spaces represent every

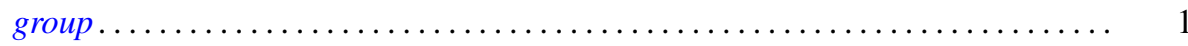

Richard Neal Ball, Full convex l-subgroups and the existence of $a^{*}$-closures of

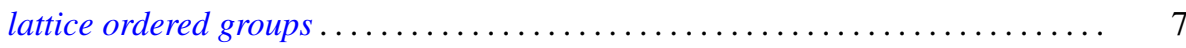

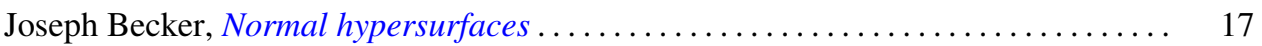

Gerald A. Beer, Starshaped sets and the Hausdorff metric . . . . . . . . . . . . . 21

Dennis Dale Berkey and Alan Cecil Lazer, Linear differential systems with

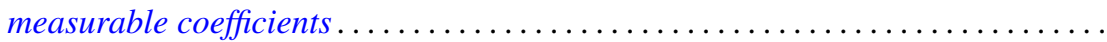

Harald Boehme, Glättungen von Abbildungen 3-dimensionaler

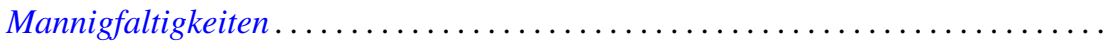

Stephen LaVern Campbell, Linear operators for which $T^{*} T$ and $T+T^{*}$

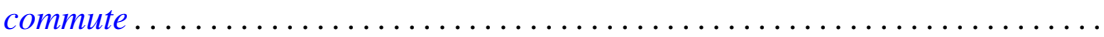

H. P. Dikshit and Arun Kumar, Absolute summability of Fourier series with

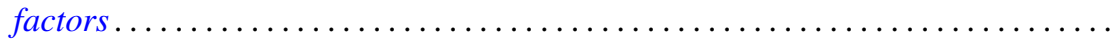

Andrew George Earnest and John Sollion Hsia, Spinor norms of local integral

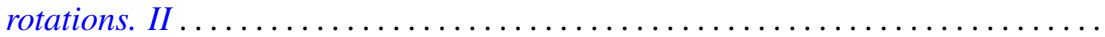

Erik Maurice Ellentuck, Semigroups, Horn sentences and isolic structures .........

Ingrid Fotino, Generalized convolution ring of arithmetic functions . . . . . . . . . . .

Michael Randy Gabel, Lower bounds on the stable range of polynomial rings .......

Fergus John Gaines, Kato-Taussky-Wielandt commutator relations and

characteristic curves

Theodore William Gamelin, The polynomial hulls of certain subsets of $C^{2}$

R. J. Gazik and Darrell Conley Kent, Coarse uniform convergence spaces. . .

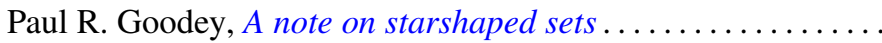

Eloise A. Hamann, On power-invariance

M. Jayachandran and M. Rajagopalan, Scattered compactification for $N \cup\{P\}$. . .

V. Karunakaran, Certain classes of regular univalent functions .

John Cronan Kieffer, A ratio limit theorem for a strongly subadditive set function in a locally compact amenable group .................

Siu Kwong Lo and Harald G. Niederreiter, Banach-Buck measure, density, and uniform distribution in rings of algebraic integers ........

Harold W. Martin, Contractibility of topological spaces onto metric spaces ....

Harold W. Martin, Local connectedness in developable spaces .

A. Meir and John W. Moon, Relations between packing and covering numbers of a tree.

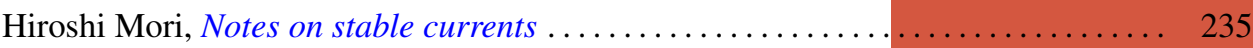

Donald J. Newman and I. J. Schoenberg, Splines and the logarithmic function . . . . 241

M. Ann Piech, Locality of the number of particles operator....

Fred Richman, The constructive theory of $K T$-modules .......

Gerard Sierksma, Carathéodory and Helly-numbers of

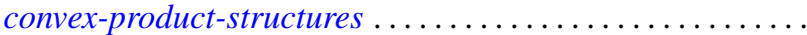

Raymond Earl Smithson, Subcontinuity for multifunctions .... . . 